Revue internationale P.M.E.

\title{
La gestion des ressources humaines dans les PME en hypercroissance \\ Frein ou levier?
}

Human resource management in high growth small and medium-sized firms

Threat or opportunity?

La gestión de los recursos humanos en las pequeñas y medianas empresas con gran crecimiento

\section{Freno o palanca?}

\section{Amaury Grimand}

Volume 26, numéro 3-4, 2013

URI : https://id.erudit.org/iderudit/1024520ar

DOI : https://doi.org/10.7202/1024520ar

Aller au sommaire du numéro

Éditeur(s)

Editions EMS - In Quarto SARL

ISSN

0776-5436 (imprimé)

1918-9699 (numérique)

Découvrir la revue

Citer cet article

Grimand, A. (2013). La gestion des ressources humaines dans les PME en hypercroissance : frein ou levier ? Revue internationale P.M.E., 26(3-4), 89-115. https://doi.org/10.7202/1024520ar
Résumé de l'article

Les PME en hypercroissance ou " gazelles " sont des acteurs essentiels de la création d'emplois et de la dynamisation des territoires. Si la recherche a surtout privilégié l'analyse des caractéristiques structurantes des gazelles et des déterminants de leur croissance, peu d'attention a été accordée à la gestion des ressources humaines dans ce type d'entreprise. Sur la base d'une étude comparative de cas, cette contribution analyse les effets structurants de la dynamique d'hypercroissance sur la fonction ressources humaines et les tensions qui l'accompagnent. La discussion souligne le rôle de l'ambidextrie organisationnelle, temporelle, contextuelle et de réseau comme leviers de régulation de ces tensions. 


\title{
La gestion des ressources humaines dans les PME en hypercroissance : frein ou levier?
}

\begin{abstract}
Amaury GRIMAND
Amaury GRIMAND est professeur des Universités en sciences de gestion, à l'IAE de Poitiers où il enseigne la gestion des ressources humaines et le management stratégique. Il dirige le CEREGE, centre de recherche en gestion de l'Université de Poitiers. Ses thèmes de recherche se centrent sur la gestion des compétences, l'appropriation des outils de gestion, la dynamique des processus de changement, les PME en hypercroissance.

IAE, Université de Poitiers

Laboratoire CEREGE (EA 1722)

20, rue Guillaume VII Le Troubadour

BP 639 - 86022 POITIERS CEDEX

agrimand@iae.univ-poitiers.fr
\end{abstract}

\section{RÉSUME}

Les PME en hypercroissance ou "gazelles" sont des acteurs essentiels de la création d'emplois et de la dynamisation des territoires. Si la recherche a surtout privilégié l'analyse des caractéristiques structurantes des gazelles et des déterminants de leur croissance, peu d'attention a été accordée à la gestion des ressources humaines dans ce type d'entreprise. Sur la base d'une étude comparative de cas, cette contribution analyse les effets structurants de la dynamique d'hypercroissance sur la fonction ressources humaines et les tensions qui laccompagnent. La discussion souligne le rôle de l'ambidextrie organisationnelle, temporelle, contextuelle et de réseau comme leviers de régulation de ces tensions.

MOTS CLÉS

PME, Hypercroissance, Gestion des ressources humaines, Tensions, Ambidextrie

\section{Human resource management in high growth small and medium-sized firms : threat or opportunity?}

\section{SUMMARY}

High growth small and medium-sized firms, usually known as "gazelles", are key actors of job creation and revitalization of territories. Most of the focus of academic research has been on the gazelles' characteristics and the factors of their growth, but little attention has been paid to the nature of human resources management (HRM) practices in such organizations. From a comparative case study, this paper analyzes the consequences of the growth dynamics on human resource management and the induced tensions. It underlines the importance of organizational, temporal, contextual and relational ambidexterity in dealing with these tensions.

\section{KEYWORDS}

Small and medium sized firms, High growth, Human resource management.

Tensions, Ambidexterity 


\title{
La gestión de los recursos humanos en las pequeñas y medianas empresas con gran crecimiento : freno o palanca?
}

\begin{abstract}
RESUMEN
Las pequeñas y medianas empresas con gran crecimiento, llamadas "gacelas", son los actores mayores de la creación de empleo y del dinamismo de un territorio. Si la investigación ha tratado de las caracteristicas estructurantes de estas "gacelas", y los determinentes de su crecimiento, poco valor ha sido dado a la gestión de los recursos humanos en este tipo de empresa. A partir de un estudio comparativo de casos, esta contribucion analiza los efectos de la dinamica de gran crecimiento sobre la competencia recursos humanos y las tensiónes que las conlleva. La discusión desarolla varias pistas sobre la resolución de aquellas tensiónes.
\end{abstract}

Palabras clave

Pequeñas y medianas empresas, Gran crecimiento, Gestión de los recursos humanos Tensiones, Organización ambidiestra

\section{Introduction}

Les PME en hypercroissance, communément dénommées "gazelles ", reçoivent aujourd'hui une attention accrue de la part de la communauté académique. Pour autant, les recherches traitant des effets structurants du phénomène d'hypercroissance sur les pratiques managériales restent rares. Du moins éprouvent-elles une difficulté à dépasser les approches fondées sur les stades de croissance dont la validité empirique est pourtant loin dêtre établie. Cette contribution entend précisément traiter de la relation entre hypercroissance et pratiques managériales à travers le prisme de la gestion des ressources humaines. Le choix d'une entrée privilégiée par la fonction $\mathrm{RH}$ tient à ce que les ressources humaines soient souvent évoquées comme une barrière importante à la croissance (Quader, 2007).

Cet article s'articule ainsi autour de la problématique centrale suivante : quelles tensions et paradoxes fait peser la dynamique d'hypercroissance sur la gestion des ressources humaines? Les effets structurants de la dynamique d'hypercroissance sur la GRH seront ici analysés tant du point de vue de son statut que de la philosophie gestionnaire qui l'anime ou des pratiques dans lesquelles elle s'incarne. Dans cette perspective, les questionnements sont multiples : quelles problématiques ressources humaines dominent dans les PME en hypercroissance? La dynamique d'hypercroissance induit-elle une transition observable de modèles de GRH pour des PME dont les pratiques de GRH empruntent souvent au modèle arbitraire (Nizet et Pichault, 2000) ? Plus fondamentalement, la relation entre GRH et hypercroissance doit-elle uniquement être appréhendée sous l'angle des tensions qu'elle suscite? Dans quelle mesure la GRH constitue-t-elle un moteur de la dynamique même d'hypercroissance ? Cette contribution appelle également un certain nombre de questionnements théoriques. Ainsi, l'approche 
par les stades de croissance est-elle réellement pertinente pour saisir la relation dynamique qui s'instaure entre GRH et hypercroissance? Une approche par les tensions ou le management paradoxal ne serait-elle pas davantage opportune?

La revue de littérature permet d'interroger dans une première partie le statut et les caractéristiques de la GRH dans les PME en hypercroissance. Nous mobilisons alors dans une deuxième partie une étude comparative de la trajectoire de trois « gazelles » afin de saisir les modalités de l'articulation entre GRH et hypercroissance. La discussion permet de revenir sur la nature des tensions induites par l'hypercroissance sur la gestion des ressources humaines ainsi que sur les modalités de leur régulation.

\section{LA GRH DANS LES PME EN HYPERCROISSANCE : CONTRAINTE OU OPPORTUNITÉ?}

Dans cette section, nous revenons dans un premier temps sur la caractérisation du phénomène d'hypercroissance et les principes structurants des " gazelles " (1.1.). Nous étudions alors la place singulière de la GRH dans les PME en hypercroissance, en nous efforçant d’en repérer les problématiques les plus prégnantes et les pratiques dans lesquelles elle s'incarne (1.2.). Nous questionnons l'hypothèse selon laquelle le phénomène d'hypercroissance amènerait une transition de modèles de GRH (1.3.). Nous proposons enfin une lecture alternative de la relation entre hypercroissance et GRH, en invitant à l'aborder non pas exclusivement sous l'angle des tensions, mais en soulignant le rôle moteur des ressources humaines dans la dynamique même d'hypercroissance (1.4.).

\subsection{Le phénomène d'hypercroissance et les caractéristiques structurantes des gazelles}

Rares sont les PME qui survivent plus de dix ans après leur création. Plus rares encore sont celles qui développent une croissance rapide et parviennent à soutenir ce rythme de croissance. Ces PME, que certains qualifient de " gazelles ", sont des acteurs essentiels de la croissance de l'emploi et de la revitalisation des territoires. La France, en l'espèce, souffrirait d'un déficit de gazelles au regard d'autres contextes nationaux (Betbèze et Saint-Étienne, 2006). Dans le cadre de cette contribution, nous définissons les PME en hypercroissance à partir d'un critère de croissance annuelle de leur chiffre d'affaires supérieur à $20 \%$ durant au moins quatre années consécutives, nous inscrivant en cela dans la continuité des principales études sur les gazelles (Julien et al., 2002 ; Nicholls-Nixon, 2005). Cette hypercroissance amène en quelques années un doublement de la taille de ces PME (Mustar, 2002 ; Picard, 2006). Si l’on retient fréquemment la définition fondatrice de la croissance posée par Penrose (1959) qui l'envisage comme un "processus évolutionniste basé sur un accroissement cumulatif des connaissances collectives ", son objectivation a fait l'objet de représentations différentes dans la littérature. La croissance du volume des ventes et des effectifs ou leur variation en taux demeure néanmoins les mesures les plus communément admises (Delmar, Davidsson et Gartner, 2003 ; Janssen, 2005). 
S’agissant des gazelles, plusieurs travaux se sont attachés à repérer les caractéristiques de ces PME à croissance rapide. On évoquera notamment à la suite de Julien (2001) :

- Un dirigeant mieux éduqué, témoignant d’une connaissance aiguë du secteur d’activité : doté d'un important capital social, le dirigeant entrepreneur de gazelle envisage l'exercice du leadership comme une fonction nécessairement partagée. La structuration d'une véritable équipe de direction apparait à cet égard comme un déterminant important de la trajectoire ultérieure de croissance ;

- Le choix délibéré de stratégies de différenciation fondées sur un standard élevé de qualité, une grande proximité avec la clientèle, l'instauration de relations durables et partenariales avec les autres parties prenantes. La proximité et l'interaction avec le client permettent de recevoir les impulsions du marché et alimentent la dynamique d'innovation ;

- Une philosophie gestionnaire visant à développer une organisation apprenante : les gazelles apparaissent ainsi dotées de modes d'organisation flexibles. Cette capacité de réorganisation permanente permet aux gazelles de mieux adapter leurs compétences aux nouvelles conditions de marché ;

- Une inscription soutenue des dirigeants et responsables dans des réseaux professionnels et réseaux de veille, et une capacité à mobiliser des ressources externes.

\subsection{La place de la gestion des ressources humaines dans les PME en hypercroissance}

La fonction ressources humaines dans les gazelles porte la marque du contexte de PME. Son absence de structuration, la faiblesse de l'expertise interne $\mathrm{RH}$, la prégnance de l'informel dans la conception et le déploiement des politiques de GRH, sont ainsi fréquemment mises en exergue. La fonction RH en PME apparaît de fait souvent réduite à sa dimension d'expertise administrative ou de conformation aux obligations légales (Mahé de Boislandelle, 1988). Les caractéristiques personnelles du dirigeant, ses croyances et représentations, ses motivations, son parcours, ses modes de relation à autrui, la philosophie managériale qui est la sienne, influencent fortement la structuration de la politique de GRH (Parlier, 2006). Tout en prenant acte de l'existence de multiples facteurs de contingence, de nombreux auteurs s'accordent à reconnaître une spécificité de l'instrumentation et des pratiques de GRH en PME (Parlier, 2006 ; Louart et Vilette, 2010) dont les traits saillants sont résumés ci-après :

- Recrutement centré sur l'activation d'un réseau relationnel et le recours à la cooptation impliquant une faible diversité des canaux de recrutement et outils de sélection mobilisés ;

- Ampleur modeste des investissements en formation continue, souvent par crainte de voir le salarié monnayer la qualification acquise ailleurs ;

- Pratiques salariales en deçà des standards du marché et faible poids des périphériques de rémunération. Léquité interne des rémunérations n'est pas toujours assurée ; 
- Faiblesse des démarches de gestion prévisionnelle des emplois et des compétences;

- $(\mathrm{GPEC})^{1}$, souvent circonscrites à l'anticipation des mouvements de personnel, mais peu disertes sur l’analyse prospective des métiers et des compétences requises ;

- Faiblesse de la dynamique de négociation collective et de la représentation syndicale. Cela ne traduit pas fatalement un climat social dégradé, mais est parfois la conséquence de relations directes et de proximité entre salariés et dirigeants (Jaouen et Torrès, 2008);

- Poids de la dimension affective dans les relations de travail et de la dimension symbolique de l'organisation, cette dernière s'articulant autour des mythes fondateurs de l'entreprise (la création de l'organisation, le dirigeant, les récits d'échecs et de succès).

Ces caractéristiques ne sont pas sans évoquer le modèle " arbitraire » de GRH développé par Nizet et Pichault (2000). La centralisation de la politique RH, la difficulté à garantir l'équité interne de certaines décisions sociales, faute de processus $\mathrm{RH}$ structurés, induisent un risque élevé de dérive des coûts cachés (notamment une augmentation des phénomènes d'absentéisme et de rotation du personnel). Ce modèle arbitraire est susceptible de constituer un handicap durable dans la mise en ouvre de stratégies de différenciation, la dégradation du climat social pouvant notamment avoir des effets néfastes sur la qualité de service.

Les caractéristiques des PME conduisent par ailleurs à exacerber les problématiques RH, et ce d'autant que les décisions sociales y ont un effet multiplicateur, un impact considérable à l'échelle d'une entreprise de taille réduite. C'est vrai en particulier des décisions de mobilité, de recrutement, de réorganisation ou bien encore des conséquences induites par l'absentéisme et la rotation du personnel (Bootz, Schenk et Sonntag, 2010). S’agissant du cas spécifique des PME en hypercroissance, celles-ci s'affrontent à cinq tensions majeures en matière sociale :

- Les difficultés de recrutement sont récurrentes du fait d'une attractivité plus réduite en termes d'emploi et d'une maîtrise parfois déficiente des canaux et outils de recrutement (Paradas, 2010);

- La fidélisation des salariés s'avère complexe, du fait d'un manque de ressources et d'un déficit de légitimité. Elle constitue pourtant un enjeu majeur en raison de l'adoption privilégiée de stratégies de différenciation;

- De façon générale, Defélix et Retour (2003a, b) notent que les «difficultés de compétences » constituent une problématique majeure des PME à croissance rapide, qu'il s'agisse de l'acquisition des compétences (recrutement, intégration, gestion de la marque-employeur...), de leur stimulation et développement (formation, mobilité, mise en œuvre

1 Nous reprenons ici à notre compte la définition de la GPEC donnée par Dejoux (2008, p. 60) qui en fait un dispositif de GRH destiné à « lier les besoins RH à la stratégie de l'entreprise; anticiper les besoins en emplois et compétences et les comparer aux ressources humaines actuelles; dialoguer avec les partenaires sociaux sur les moyens à mettre en cuvre (formation, bilan de compétences, mobilité, VAE) pour accompagner collectivement et individuellement le développement des compétences nécessaires à la pérennité de l'emploi et de l'entreprise ". 
d’organisations « apprenantes »...) ou bien encore de leur fidélisation (reconnaissance des compétences acquises, pratiques de responsabilisation, culture organisationnelle...);

- Les PME en hypercroissance s'affrontent à la nécessité de changer rapidement de culture et/ ou de modèle de GRH lors des phases de réorientation stratégique (Louart et Vilette, 2010). De fait, le modèle arbitraire de GRH apparaît difficilement compatible avec le maintien d'une dynamique soutenue de croissance, imposant une rationalisation à minima des principaux processus RH ainsi qu'un partage de la fonction RH avec l'encadrement intermédiaire ;

- Si l'instauration et le maintien d'une culture organisationnelle forte apparaissent comme un mécanisme d'intégration essentiel face à une dynamique de croissance qui a plutôt pour effet d'accentuer la différenciation des unités, l'accroissement de la complexité organisationnelle, consécutivement à la croissance, peut mettre à mal le modèle culturel fondateur.

\subsection{L'hypercroissance, moteur d'une transition de modèles de GRH ?}

Dès 1988, Mahé de Boislandelle avançait l'hypothèse selon laquelle les modalités de prise en charge de la fonction $\mathrm{RH}$ variaient avec la taille. Dans une étude comparative de cas portant sur les interactions entre stratégie et GRH dans l'industrie du jeu vidéo, Picq et Autier (2002) montrent que chaque palier de croissance peut être associé à une configuration de pratiques de GRH, cohérentes entre elles d'une part, mais aussi cohérentes avec la stratégie et les modes d'organisation internes d'autre part. On reconnaîtra sans peine dans cette perspective les préceptes des approches fondées sur les stades de croissance qui ont fait l'objet depuis le modèle fondateur de Greiner (1972), d'une quantité de théorisations dans la littérature. L'hypothèse implicite qui fonde ces modèles est qu'il est possible de résumer la trajectoire de croissance en une succession linéaire de stades, chacun impliquant une tension managériale dominante. Transposée à la GRH, cette perspective induit l'idée d'une transition de modèles de GRH (entendus ici comme des configurations cohérentes de politiques et de pratiques de GRH) générée par l'hypercroissance. Pour autant, et bien quayant reçu un large écho dans la littérature, il n’est pas sûr que les approches fondées sur les stades de croissance soient de nature à éclairer la relation entre GRH et hypercroissance. En effet, dans la lignée des arguments avancés par Levie et Lichtenstein (2010), à partir de l'étude de 104 modèles fondés sur les stades de croissance et publiés entre 1962 et 2006, on pointera :

- L'absence de définition convaincante dans les travaux de ce qu'est un stade de croissance ;

- L'absence de consensus sur le nombre de stades de croissance;

- Une affirmation de la capacité prédictive de ces modèles qui souffre cruellement de l'absence de validation empirique ;

- Un biais d'anthropomorphisme qui consiste à établir une analogie entre la croissance de l'entreprise et celle de l'enfant devenant adolescent puis adulte.

Dès lors, dans les cas étudiés, qu’en est-il des stades de croissance ? Obéissent-ils à une séquence prédéterminée ? Peut-on associer à cette trajectoire d'hypercroissance une transition observable de modèles de GRH ? 


\subsection{Un regard alternatif : les ressources humaines comme moteur de la croissance}

La littérature dédiée à la relation entre croissance et GRH a tendance à appréhender celle-ci exclusivement en termes d'impact du phénomène d'hypercroissance sur la fonction RH. La $\mathrm{GRH}$, dans cette optique, est analysée comme une variable dépendante ; elle est façonnée directement par la dynamique d'hypercroissance. C'est ici une conception dominante de la GRH comme source de blocage à la croissance qui s'impose.

Si la configuration des politiques et pratiques de GRH peut indubitablement obérer la dynamique de croissance, on aurait tort de résumer la relation GRH - croissance à ce seul prisme. Une lecture alternative de cette relation, moins répandue dans la littérature, consiste à envisager que les ressources humaines puissent contribuer à soutenir la dynamique de croissance. Cette relation n'est pas directe, mais opère via la médiation de l'avantage concurrentiel et la contribution des ressources humaines aux stratégies de différenciation, caractéristiques des gazelles. De ce point de vue, les approches fondées sur les ressources, dont on sait l'écho qu'elles ont eu dans le champ du management stratégique (Barney, 1991), ont été transposées avec succès en GRH (Wright, McMahan et McWilliams, 1994). Dans ce cadre, la relation entre ressources humaines et création de valeur est envisagée comme une « relation médiatisée par le développement préalable d'un avantage concurrentiel construit sur des processus ressources humaines qui ont les caractéristiques de ressources valorisantes, spécifiques, non imitables et non substituables » (Besseyre des Horts, 2002). Lado et Wilson (1994) prolongent ce raisonnement en suggérant que les politiques et pratiques de GRH contribuent à l'avantage concurrentiel par le développement de compétences spécifiques à la firme, inscrites dans sa culture et son histoire, la création de relations sociales complexes et le développement de processus organisationnels à forte composante tacite incorporée.

Dans le prolongement de la théorie des ressources, Barringer, Jones et Lewis (1998), à travers une étude portant sur 70 entreprises en hypercroissance, mettent en relief le rôle clé de la GRH dans la capacité à gérer la croissance :

- La promotion interne est encouragée afin de réduire les coûts d'intégration ;

- La culture organisationnelle et le charisme du dirigeant permettent de préserver la cohérence de l'organisation, mise à mal par la croissance ;

-L'instauration de dispositifs incitatifs permet d’associer les salariés aux fruits de la croissance ;

- Les pratiques de responsabilisation des salariés et la construction dorganisations « apprenantes » sont simultanément un levier de flexibilité et de fidélisation des salariés ;

- Pour sa part, Parlier (2010) suggère que seules les PME en croissance adoptent une gestion par les compétences, faisant de la compétence un concept pivot autour duquel s'articulent le système de GRH, mais aussi les pratiques d'organisation (accent porté sur la polyvalence, l'autonomie, le travail en mode projet, etc.);

- Enfin, les gazelles apparaissent dotées d'une forte capacité d'apprentissage organisationnel à travers laquelle elles infléchissent leurs routines, initient des bifurcations stratégiques, saisissent des opportunités afin de soutenir la croissance (Chanut-Guieu et Guieu, 2011). 
Au terme de cette revue de littérature, si nous avons pu mettre en évidence les caractéristiques structurantes des gazelles d'un point de vue stratégique ainsi que la spécificité des problématiques et pratiques de GRH en leur sein, les champs de l'hypercroissance et de la GRH apparaissent encore largement désarticulés dans la production académique. C’est à cet effort d’articulation que l'étude comparative de cas qui suit invite.

\section{LES MODALITÉS DE L'ARTICULATION ENTRE GRH ET HYPERCROISSANCE : UNE ÉTUDE COMPARATIVE DE LA TRAJECTOIRE DE TROIS GAZELLES}

\subsection{Méthodologie}

La recherche réalisée repose sur une étude comparative de cas, qui est elle-même partie prenante d'un projet de recherche collectif plus vaste intitulé " Les entreprises moyennes en hypercroissance : trajectoires et temporalités ", initié avec le soutien de l'ANR (Agence nationale de la recherche, France) sur la période 2007-2010. La restitution qui en est proposée dans le cadre de cette contribution repose sur l'analyse de trois gazelles oeuvrant dans des contextes d'activités différents : nettoyage et propreté, mise en marché de produits alimentaires bio, fabrication de thermocollants. Les études de cas sont qualifiées de longitudinales rétrospectives, à même de restituer la dynamique de croissance dans ses interactions avec la fonction ressources humaines. La démarche de recherche mobilise un cadre d'analyse contextualiste (Pettigrew, 1985) souvent convoqué pour l'étude du changement organisationnel, ce dernier étant appréhendé comme le fruit de trois composantes en interaction :

- Le contexte, en l'occurrence la place de l'environnement, mais aussi les effets induits par les caractéristiques internes de l'organisation (culture, niveau de qualification du personnel, organisation du travail...) sur la trajectoire de croissance ;

- Le contenu du changement, soit l'évolution simultanée de la croissance et de la fonction ressources humaines;

- Les processus à l'œuvre, notamment la façon dont les acteurs et l'organisation renouvellent leurs cadres de pensée et modes d'action au fur et à mesure de la croissance.

La recherche s'inscrit dans une posture interprétativiste accordant une attention équilibrée aux actions et événements, mais aussi aux significations qu’en développent les acteurs.

La collecte des données a permis de capitaliser sur un matériau empirique constitué de 19 entretiens ${ }^{2}$ centrés sur des acteurs majeurs de la croissance des entreprises étudiées. Les entretiens ont été réalisés sur la base d'un guide comportant une base commune, relative au phénomène d'hypercroissance et à ses implications, et un volet de questionnements spécifiques, en fonction du profil de la personne interviewée. La trame commune interrogeait en particu-

2 Les acteurs rencontrés ainsi que les principales caractéristiques des entreprises composant létude comparative de cas sont repris dans l'annexe 1 . 
lier la dynamique de croissance dans ses différentes phases, les motivations à la croissance, la stratégie déployée, l'environnement et les marchés, les structures de gouvernance, l'accès aux sources de financement, le rôle des fonctions support dans la trajectoire de croissance. Les entretiens ont été systématiquement enregistrés et retranscrits.

Afin de limiter les biais de rationalisation a posteriori, inhérents à toute démarche rétrospective, les interviews ont été croisées avec de multiples données secondaires (rapports annuels, revue de presse, etc.). L'insistance portée dans les entretiens sur les changements majeurs a été utilisée pour atténuer les limites des capacités de mémorisation des acteurs interviewés.

La démarche de recherche adoptée peut être qualifiée d' " abductive ", au sens où elle développe, à partir de l'observation, des conjectures qu'elle s'efforce ensuite de tester et de discuter (Koenig, 1993). Elle est marquée, de fait, par des itérations successives entre les cadres théoriques convoqués et l'analyse des matériaux empiriques collectés (Charreire et Durieux, 2003).

L’analyse des données a mobilisé pour l'essentiel deux étapes :

- Une première étape visant à construire une narration des trois cas étudiés afin de rendre compte de la relation dynamique qui s'instaure entre GRH et hypercroissance ;

- Une deuxième étape consistant en une analyse thématique des données collectées afin de produire un schéma d'interprétation de cette relation entre GRH et hypercroissance, d'en éclairer les différentes dimensions. L'identification des thèmes s'est opérée en amont sur la base d'un double corpus théorique, relatif à l'hypercroissance d'une part, et la GRH en PME d'autre part. Des thèmes émergents ont pu néanmoins être intégrés lors de la phase de codage.

L’analyse thématique réalisée permet de restituer la relation dynamique existant entre le phénomène d'hypercroissance et le système de GRH. Cette relation paraît ainsi s'inscrire dans un triple registre : 1) celui de la vision ressources humaines (impact du phénomène d'hypercroissance sur les rôles dévolus à la fonction $\mathrm{RH}$ et son degré de structuration) ;2) celui de l'instrumentation de GRH (effets structurants de la croissance sur les outils et les pratiques de GRH) ; 3) celui de la contribution des ressources humaines à l'avantage concurrentiel et à la dynamique d'hypercroissance. Le tableau suivant détaille la nature du codage thématique réalisé à partir de la base d'entretiens :

Nous exposons succinctement ci-après les contours de la vision stratégique des trois entreprises considérées ainsi que les phases clés de leur trajectoire de croissance.

BIO DISTRI $^{3}$ est un des leaders européens dans la commercialisation de fruits et de légumes bio. Créée en 1987, ayant son siège social dans le Vaucluse, elle comprenait 220 salariés en 2008 et a connu une croissance annuelle moyenne de $20 \%$ sur la période 2005-2009, via notamment des opérations de croissance externe.

Trois grandes phases peuvent être distinguées dans la trajectoire de croissance de l'entreprise :

- La première, de 1987 à 1992, est marquée par la dimension militante d'un projet, à une époque où le marché du bio reste embryonnaire. La croissance est portée par la demande

3 Les noms des trois entreprises composant l'étude comparative de cas ont été changés pour des raisons de confidentialité. 
qui se structure progressivement, sans correspondre à une volonté explicite de la part du fondateur. La stratégie apparaît largement guidée par les événements extérieurs ;

- La deuxième phase, de 1992 à 2001, marque une volonté de structuration tant de l'entreprise que du marché bio. Cette volonté naît d'un événement traumatisant : une grève des transports qui bloqua l'entrepôt du Vaucluse pendant plusieurs semaines, entraînant le licenciement de quatre salariés. BIO DISTRI sattache dès cette époque à diversifier ses sources d’approvisionnement, marchés et clients ;

- La troisième phase séchelonne de 2001 à 2009. Elle engage un effort progressif de structuration d'une véritable équipe de direction dont l'acte de naissance sera officialisé en 2004. Simultanément, cette période est le point de départ de plusieurs opérations de croissance externe qui s'accéléreront suite à l'entrée d'un fonds d'investissement en 2005 qui prend alors $51 \%$ des parts.

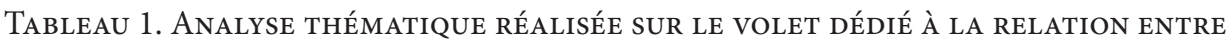 GRH ET HYPERCROISSANCE}

\begin{tabular}{|c|c|}
\hline Catégories & Thèmes \\
\hline $\begin{array}{l}\text { Hypercroissance et } \\
\text { "vision" ressources } \\
\text { humaines }\end{array}$ & $\begin{array}{l}\text { - Impact du phénomène d'hypercroissance sur le niveau de } \\
\text { structuration de la fonction RH } \\
\text { - } \quad \text { Impact du phénomène d'hypercroissance sur le degré de } \\
\text { "partage » de la fonction RH } \\
\text { - } \quad \text { Hypercroissance et transformation des rôles dévolus à la } \\
\text { fonction RH } \\
\text { - Hypercroissance et transition de modèles de GRH } \\
\text { - Hypercroissance et légitimité de la fonction RH }\end{array}$ \\
\hline $\begin{array}{l}\text { Hypercroissance et } \\
\text { instrumentalisation } \\
\text { de GRH }\end{array}$ & $\begin{array}{ll}\text { - } & \text { Impact des effets de seuil sur les pratiques et outils de GRH } \\
\text { - } & \text { Hypercroissance et cohérence interne des pratiques de GRH } \\
\text { - } & \text { Usages de la GPEC dans les PME en hypercroissance }\end{array}$ \\
\hline $\begin{array}{l}\text { Contribution de la GRH } \\
\text { à la dynamique } \\
\text { d'hypercroissance }\end{array}$ & $\begin{array}{l}\text { - } \quad \text { Hypercroissance et stratégies de rétention de la main d’œuvre } \\
\text { - } \quad \text { Caractéristiques de création de valeur, de rareté, d'imitabilité } \\
\text { imparfaite attachées aux ressources humaines } \\
\text { - } \quad \text { Alignement GRH - stratégie dans les PME en hypercroissance } \\
\text { - } \quad \text { GRH et capacité d’ambidextrie } \\
\text { - Hypercroissance et culture organisationnelle }\end{array}$ \\
\hline
\end{tabular}

L'entreprise TEX est spécialisée dans la fabrication de thermocollants pour textiles, destinés notamment au marquage pour transfert à chaud de tee-shirts sportifs. Créée en 1983, implantée à Saint-Étienne, où elle dispose d'un site de production industriel, elle œuvre pour quatre types principaux de clients que sont les distributeurs de la sérigraphie, du textile, de la signalétique et de la création graphique (communication d'entreprise).

Trois phases principales ponctuent la trajectoire de croissance de TEX :

- La première phase (1983-2005) est marquée par une stratégie progressive de développement sur le marché français. Cette période consacre l'emprise du dirigeant fondateur qui concentre dans ses mains les principales fonctions opérationnelles; 
- 2005 marque une rupture importante avec la reprise de TEX par un nouveau directeur général qui rachète l'entreprise sous la forme d'un LBO (Leveraged Buy-Out). Cet événement marque le début d'un investissement soutenu dans la gamme de produits avec la recherche de nouvelles matières et de nouveaux coloris ;

- La période 2008-2010 est marquée par des actions visant à améliorer la rentabilité via une démarche d'amélioration continue du processus de production afin d'initier des gains de productivité et de mieux maîtriser les coûts.

L'entreprise NETTOYAGE SUD-EST ${ }^{4}$, créée en 1967 à Gap, est spécialisée dans le nettoyage de bureaux, de locaux industriels et commerciaux, tout en proposant une gamme de services associés. Acteur régional important, NETTOYAGE SUD-EST a connu une trajectoire de croissance fortement imbriquée avec une histoire familiale :

- De 1967 à 1984, le fondateur, animé par la volonté de ne pas rester artisan, impulse une logique de développement local, via la création de plusieurs agences. Le cap de 100 salariés est franchi en 1984. Le développement local laissera la place de 1984 à 1993 à un développement régional avec notamment un essor soutenu sur la région Rhône-Alpes ;

- 1999 marque un point d'inflexion majeur avec le franchissement du cap de 500 salariés, l'obtention de la certification ISO et un drame accidentel qui voit la disparition de deux des plus proches collaborateurs du père fondateur. Cet épisode va accélérer la montée en puissance du fils dans le groupe, qui sera actée formellement par sa nomination en tant que directeur général en 2007 ;

- La période de 2000 à 2005 signale l'entrée dans un régime d'hypercroissance avec un quasidoublement des effectifs jusquà atteindre le seuil de 900 salariés (les prévisions formulées à horizon 2010 sont ainsi atteintes dès 2005 !) ;

- La période postérieure à 2005 marque un certain ralentissement de la croissance.

On ajoutera que pour l'ensemble des dirigeants interviewés, c'est moins la croissance per se qui importe que la recherche d'une croissance rentable : "La croissance pour la croissance, ça ne veut rien dire. Moi, quand je parle de croissance, c'est de croissance rentable. Cette croissance rentable est forcément associée à une augmentation de la productivité et une baisse des coûts » (D-G, TEX).

\subsection{La croissance comme rencontre entre une vision stratégique et un contexte favorable}

Pour Gasse (1996), le phénomène de croissance résulte de la rencontre entre une vision stratégique, un potentiel de réalisation de cette vision (lié à la capacité de l'entreprise à combiner, intégrer des ressources différentes), et un contexte favorable. Cette interprétation de la croissance peut être transposée aux trois entreprises fondant l'étude comparative de cas.

4 L'entreprise NETTOYAGE SUD-EST, composée aujourd'hui de 1300 salariés (617 en équivalent temps plein), dépasse largement les seuils usuellement retenus pour qualifier une PME. Toutefois, il nous semblait opportun dans l'échantillon d'avoir un cas d'entreprise ayant "digéré " avec succès la phase d'hypercroissance tout en étant en mesure de reconstituer sa trajectoire de façon rétrospective. 
La trajectoire de croissance de chacune d'entre elles est en effet inspirée par une vision stratégique clairement affirmée dont les fondements ne sont pas sans rappeler l'analyse développée par Julien (2001) quant aux caractéristiques structurantes des gazelles.

\subsubsection{LA PRÉGNANCE DES STRATÉGIES DE DIFFÉRENCIATION}

Les trois entreprises considérées ont opté pour des stratégies de différenciation fondées sur l'innovation, une gestion personnalisée de la relation client, un standard élevé de qualité. TEX se démarque ainsi de la concurrence en proposant l'une des offres produits les plus étendues du marché, avec notamment le développement d'une gamme de pastels pour le marquage sport féminin et la création d'une gamme écologique, bio-sourcée à plus de $50 \%$. BIO DISTRI se différencie des autres metteurs en marché de fruits et de légumes bio par un investissement soutenu aux différents stades de la filière. En amont, l'entreprise apporte un soutien aux producteurs dans un registre technique (conseils dans les processus de certification bio, choix des variétés, assistance par des agronomes indépendants, etc.) et dans un registre financier (avances sur récoltes). En aval, l'entreprise accompagne ses clients tant dans la conception des actions marketing, que la mise en avant des produits ou bien encore le conseil au consommateur.

NETTOYAGE SUD-EST, pour sa part, a développé une gamme de services associés (nettoyage de hottes de cuisine, dératisation, pose de moquettes, etc.) afin d'accentuer sa stratégie de différenciation et privilégier un maillage des petites villes, là où les acteurs nationaux sont moins présents. Dans tous les cas de figure, la croissance engage simultanément une diversification du marché et une expansion géographique.

\subsubsection{LA RECHERCHE D'UNE RELATION DE PROXIMITÉ AVEC LES CLIENTS}

La recherche de proximité avec la clientèle est un trait transversal aux trois entreprises étudiées comme en attestent les compte rendus suivants :

- "Quand on fait du commerce avec cet état d'esprit, on crée une cuvre commune. Lorsque je voyageais, jétais reçu chez mes clients et eux installaient leur caravane chez moi quand ils venaient en Provence. Avec les producteurs, les échanges sont amicaux, nos liens sont personnels $»(\mathrm{P} . \mathrm{D}-\mathrm{G}$, dossier de presse, 2008);

- «On existe par notre qualité, notre proximité, ça c’est important. Notre réactivité, c’est un peu notre point fort : la réactivité, la proximité, le sérieux, le suivi " (Directeur régional, NETTOYAGE SUD-EST);

- « Nos clients grossistes, ils ont besoin aussi de présenter les produits; donc, parfois on les soutient dans leurs salons " (DRH, TEX).

La proximité avec le marché est appréhendée par les acteurs interviewés comme un moyen d'en repérer les signaux faibles et d’en saisir les opportunités. Les contacts directs avec les clients apparaissent, de fait, comme un substitut à la réalisation détudes marketing. Cette proximité est également analysée comme un levier de fidélisation de la clientèle et de mise en cohérence entre image interne et image externe (elle fait écho à celle entre les dirigeants, l'encadrement et les salariés). 


\subsubsection{UN USAgE SOUPLE DES DÉMARCHES DE PLANification STRATÉGIQUE}

Dans les trois entreprises considérées, les dirigeants ne semblent pas accorder beaucoup de crédit aux démarches de planification stratégique, qui, de fait, font l'objet d'un usage pour le moins flexible : "L'adaptation c'est parfois mieux que la stratégie " (D-G NETTOYAGE SUD-EST). Il semble même que les acteurs de BIO DISTRI en fassent un usage purement rhétorique, symbolique, à des fins de légitimation de l'action, notamment auprès des actionnaires de l'entreprise :

- " Je crois beaucoup plus dans les événements extérieurs que dans les business plan. Les plan d'affaires sont toujours des choses assez rigolotes. On en a besoin parce que c'est une espèce de guide, mais la réalité est tout à fait ailleurs »(P.D-G, BIO DISTRI);

- "Les plans à 5 ans, on les construit parce qu'il faut qu'on les construise. Parce qu'on a en face de nous des gens qui sont habitués à voir des plans d'affaires à 5 ans. Et évidemment, on les construit de façon à ce qu'ils soient articulés logiquement et bien crédibles " (Contrôleur de gestion, BIO DISTRI).

Cet usage modéré de la planification stratégique semble également traduire un horizon de réflexion relativement borné :

"La banque, si vous lui proposez un investissement de 800000 euros et lui dites que ce sera rentable dans six ans, ils s'en foutent. Donc, si vous voulez, on a souvent l'obligation de réflexion sur la stratégie à court terme. Court terme c'est-à-dire un an, peut être du moyen terme à 3 ans, mais c'est tout » (D-G, TEX).

\subsubsection{LA VOLONTÉ D’APPARAÎTRE COMME UN ACTEUR INSTITUTIONNEL À PART ENTIÈRE}

La volonté d'apparaître comme un acteur institutionnel à part entière singularise les trois gazelles étudiées. C’est selon le P.D-G de BIO DISTRI l'une des motivations majeures à la croissance, l'atteinte d'une taille critique minimale étant de son point de vue indispensable pour prétendre orienter, peser sur les règles du jeu à l'intérieur du marché : "Il faut qu’on occupe une position sur le marché qui soit suffisamment forte pour essayer de construire un marché bio indépendant du marché conventionnel ». La croissance pour ces entreprises trouve ainsi sa justification dans la volonté affirmée d'imprimer fortement leur marque personnelle sur les tendances et la structuration du marché. Cette volonté trouve un point d'appui dans l'investissement conséquent des dirigeants et des principaux responsables des entreprises étudiées dans de multiples réseaux. On note ainsi une présence active des responsables de BIO DISTRI dans les différentes institutions du domaine réglementaire : Commission européenne, ministère de l'Agriculture, Comité de certification EcoCert, Présidence du comité bio de l'interprofession des fruits et légumes (Interfel). Selon le P.D-G, cette démarche vise fondamentalement à " améliorer la lisibilité du marché » tout en "luttant contre l'arrivée de metteurs en marché conventionnels qui se convertissent au bio ". Le fondateur de NETTOYAGE SUD-EST, pour sa part, est militant patronal de longue date et administrateur de la chambre régionale des entreprises de propreté. Julien et al. (2002) soulignent l'importance des réseaux pour la croissance en tant que vecteur d'innovation ou dispositif de veille permettant d'accéder à l'information pertinente à moindre coût. 


\subsection{Les effets de la dynamique d'hypercroissance sur la structuration de la fonction RH}

\subsubsection{UNE HYPERCROISSANCE QUI INDUIT UN BESOIN DE RATIONAlisation ET UN PARTAge PLUS Aigu DE LA FONCTION RH}

De façon peu surprenante, l'hypercroissance amène un besoin de rationalisation et de structuration de la fonction RH qui, de l'aveu même des acteurs interviewés, répond d'abord à un besoin de sécurisation des risques en matière sociale. Les DRH des gazelles témoignent à cet égard d'une préoccupation marquée pour la maitrise et le respect du cadre légal : « $L a$ base qui embauche les salariés doit comprendre l'intérêt de tout ce qui est législatif. Ce n'est pas évident à gérer au niveau des contrats » (DRH - NETTOYAGE SUD-EST). Dans le cas TEX, la crainte d'une démultiplication des obligations liées au droit et au contrôle de gestion sociale ont amené ses dirigeants à circonscrire la croissance au seuil de 50 salariés. "On ne passera jamais la barre des 50 salariés. Après c'est trop lourd à gérer, on a plus de charges, plus le comité d'entreprise et les obligations légales. Quand on passe à plus de 50, les charges sont beaucoup trop fortes. Donc, tant que je pourrai rester sous les 50, je le ferai. » (D-G, TEX).

Dans les trois monographies réalisées, les DRH ont recours soit à un cabinet d’avocats spécialisés en droit social ou à un conseil juridique auprès du syndicat professionnel.

Ce besoin de rationalisation est d'autant plus aigu dans les cas où l'hypercroissance est essentiellement tirée par la croissance externe. La sous-estimation des problématiques RH à l'œuvre au sein des entités rachetées ainsi que les questions d'équité nées de la nécessité d'harmoniser des systèmes de GRH différents sont ainsi mises en avant : "Lorsque nous reprenons des structures, il est dommage de ne pas avoir fait une étude approfondie du social " (DRH NETTOYAGE SUD-EST).

Cette rationalisation de la fonction $\mathrm{RH}$ s'accompagne inévitablement au-delà du franchissement d'un seuil organisationnel critique, d'un partage plus aigu de la fonction RH avec l'encadrement, notamment intermédiaire : "Avec la croissance de l'entreprise, une seule personne ne peut gérer la fonction $R H$; il faut nécessairement qu'il y ait délégation et supervision " (DRH - NETTOYAGE SUD-EST). Dans les trois cas étudiés, cette rationalisation a néanmoins souffert d'une structuration tardive de la fonction RH et, après sa création, d'un déficit de légitimité au regard d'autres fonctions opérationnelles.

\subsubsection{L'INVESTISSEMENT EN RESSOURCES RARES, CRÉATRICES DE VALEUR}

Les trois études de cas montrent que la relation entre GRH et dynamique de croissance est loin dêtre univoque. Si indéniablement la croissance fait peser des tensions fortes sur la fonction ressources humaines, cette dernière apparaît symétriquement comme constitutive de l'avantage concurrentiel, et partant, comme un élément moteur de la croissance. En atteste notamment la volonté affirmée de BIO DISTRI de capter et de fidéliser des ressources humaines rares, créatrices de valeur. Dans cette perspective, le recrutement des commerciaux apparaît comme stratégique, l'entreprise ayant fait très tôt le choix d’être à l'export. La plupart des commerciaux recrutés témoignent ainsi d'une connaissance aiguë du secteur, étant en poste depuis 10 à 15 ans dans des entreprises de fruits et légumes (qu'elles soient sur le mar- 
ché bio ou conventionnel). Cette volonté d'investir sur des ressources rares est également à l'œuvre chez TEX, bien que les marges de manœuvre apparaissent plus limitées : "Il faudrait que l'entreprise soit plus compétente dans la chimie, dans la connaissance chimique de notre processus et dans le suivi chimique de nos produits, mais je nai pas le seuil de chiffre pour avoir quelqu'un qui ne soccupe que de ça» (D-G, TEX).

Les dirigeants des trois gazelles étudiées symbolisent eux-mêmes ces ressources rares, non seulement du fait de leurs caractéristiques personnelles, mais aussi de leur investissement dans de multiples réseaux. Cet investissement participe de la recherche de normes, de certifications, de cadres de référence, permettant aux dirigeants comme à leurs organisations d'asseoir leur légitimité à l'égard de leurs parties prenantes (clients, fournisseurs, financeurs, etc.). Dans les trois études de cas considérées, les dirigeants témoignent d'un niveau d’optimisme et de satisfaction supérieur à la moyenne, rejoignant en cela les conclusions formulées par Chanut-Guieu et Guieu (2011). Le profil des dirigeants interviewés conforte également l'analyse de Marchesnay (1991) selon laquelle les entrepreneurs attirés par la croissance ont un tropisme pour la prise de risques, tout en étant attachés à leur autonomie de gestion et au caractère discrétionnaire de leurs décisions. Leur mode de gestion, participatif et impliquant, est congruent avec l'adoption d'un modèle individualisant de GRH.

\subsubsection{L'IMPÉRIEUSE NÉCESSITÉ DE LA FIDÉLISATION}

Cet investissement dans des ressources humaines rares n’a toutefois de sens quà développer une capacité aiguë de fidélisation des salariés, les ressources humaines étant par essence volatiles et soumises à la menace de rotation du personnel. Ces stratégies de rétention de la main-d’œuvre reposent chez BIO DISTRI sur des principes clairement établis : une dominante de contrats à durée indéterminée (CDI), une dynamique de promotion interne (dont on citera ici un cas exemplaire : le directeur des achats a été successivement préparateur de commandes, responsable préparation, vendeur, chef des ventes); des pratiques salariales favorables au regard des standards du marché ; un ancrage territorial de la société ; une identification forte à la personnalité du fondateur. Les compte rendus révèlent par ailleurs l'usage de leviers de motivation et de fidélisation propres à la PME et mis en relief par Parlier (2006) : la proximité avec le dirigeant, la qualité du climat social, les opportunités de développement de la polyvalence. Pour le DAF de NETTOYAGE SUD-EST, l'investissement en amont dans la qualité du recrutement et de la formation, est un élément essentiel dans la capacité à délivrer un service de qualité : "Tout repose dans le choix de cet agent de service, dans la capacité de nos chefs déquipe à bien choisir les employés. Nos équipes doivent bien intégrer leurs agents et les former aux techniques et aux méthodes de nettoyage, mais aussi leur donner une formation commerciale de base, c'est-à-dire savoir parler à un client, ne pas avoir peur d'un client, savoir se présenter. "

Par ailleurs, la fidélisation des salariés est envisagée par les dirigeants des gazelles comme un axe majeur de fidélisation de la clientèle : «On a un chef des ventes export qui est présent chez nous depuis 12 ans et un grossiste allemand qui est un client très important pour nous et est là depuis 20 ans» (DRH - BIO DISTRI). Dans le même esprit, la qualité du climat social est analysée comme un élément structurant de la qualité de service. Pour la DRH de TEX, la fidélisation des salariés est un enjeu prioritaire compte tenu de la spécificité des compétences en production et des temps d’intégration relativement conséquents en cas de 
nouvelle embauche : "À l'atelier, ça devient de plus en plus difficile la technicité. Sur une dérouleuse, pour prendre en main la machine, vous avez une semaine de formation, mais vous nêtes vraiment opérationnel qu'au bout de 6 mois parce qu'au niveau du traitement des défauts, c'est quelque chose de très difficile». Le DAF de NETTOYAGE SUD-EST, enfin, souligne la nécessité de contenir le phénomène de rotation du personnel dans un contexte sectoriel marqué par les brutales variations d'activité et l'exigence de réactivité : "C'est un métier où la gestion des ressources humaines est prépondérante. La rotation du personnel est réellement importante. Elle se monte à plus de $50 \%$. Le problème, c'est que nous ne voyons pas nos salariés. Ils sont éclatés chez nos clients. C'est le client qui nous appelle en nous disant que le salarié est absent. Notre réactivité doit par conséquent être très forte. "

La refonte du système de rémunération de TEX participe de cette volonté de fidélisation. Elle s'est traduite par la mise en place d'un accord d'intéressement avec une répartition proportionnelle au niveau de salaire et l'instauration de primes de productivité. Au-delà de leur caractère incitatif, ces primes sont envisagées par la DRH comme un instrument de dialogue visant à inscrire les référents production dans une démarche d’amélioration continue.

Indépendamment des stratégies de rétention des salariés, les gazelles investissent dans la construction de ressources humaines qui soient difficilement imitables. De même, les pratiques qui les prennent pour objet apparaissent fortement inscrites dans le contexte social et la culture interne, ce qui voue à l'échec toute tentative de les dupliquer par la concurrence.

Le développement de modes d’organisation flexibles, centrés sur la polyvalence, procède de cette volonté de construire des barrières à l'imitation des ressources humaines. Chez TEX, l'aptitude à la polyvalence est devenue un critère explicite de recrutement et un axe prioritaire de la politique de formation : "On avait quatre salariés qui sont anciens, qui ont cette polyvalence, mais, du coup, pour les nouveaux, on essaie de les former aussi et on leur inculque cette culture. On leur dit à l'entrée qu'on veut qu'ils soient polyvalents. On a intégré ce critère dans les entretiens d'embauche»(DRH, TEX).

\subsubsection{UNE TRANSITION COMPLEXE DE MODÈLES DE GRH ET UN DÉFICIT DE COHÉRENCE INTERNE}

Si l'on en croit les approches fondées sur les stades de croissance, la dynamique d'hypercroissance s'accompagnerait d'une structuration de la fonction $\mathrm{RH}$ par paliers, chaque stade ou seuil de croissance pouvant être associé à une transition de modèles de GRH. En croisant cette perspective avec l'approche configurationelle de Nizet et Pichault (2000), il est plausible d'imaginer que l'hypercroissance accompagne la séquence suivante : modèle arbitraire de GRH $\rightarrow$ modèle objectivant $\rightarrow$ modèle individualisant.

Le modèle arbitraire de GRH, marqué par l'absence de critères explicites pour fonder les politiques ressources humaines, accompagnerait ainsi les premières phases de croissance jusqu'à ce que les besoins de rationalisation et d'harmonisation des pratiques de GRH imposent un changement de modèle. Le modèle " objectivant " prendrait alors le relais en impulsant un mouvement de standardisation des pratiques de GRH dont le déclencheur réside le plus souvent dans le dépassement d'un seuil organisationnel critique ${ }^{5}$. Par réaction à

5 On situe usuellement à 100-150 salariés le seuil à partir duquel la structuration d'une fonction RH 
l'application uniforme de critères impersonnels à la gestion des relations sociales, le modèle « individualisant » tendrait à s'imposer dans les phases ultimes de croissance en mettant en jeu des stratégies de rétention du personnel fondées sur l'individualisation des pratiques de GRH et la segmentation croissante des ressources humaines (gestion des seniors, gestion des hauts potentiels, politique diversité, etc.).

L'hypothèse d'une transition linéaire de modèles de GRH induite par l'hypercroissance n'est cependant pas vérifiée dans les trois études de cas. L’exemple de BIO DISTRI, à cet égard, est éclairant. La croissance se traduit en effet dans le cas de cette entreprise par l'adoption d'un modèle de GRH hybride, empruntant simultanément aux trois modèles de GRH précités ${ }^{6}$.

FIGURE 1. BIO DISTRI : UN MODÈLE DE GRH HYBRIDE

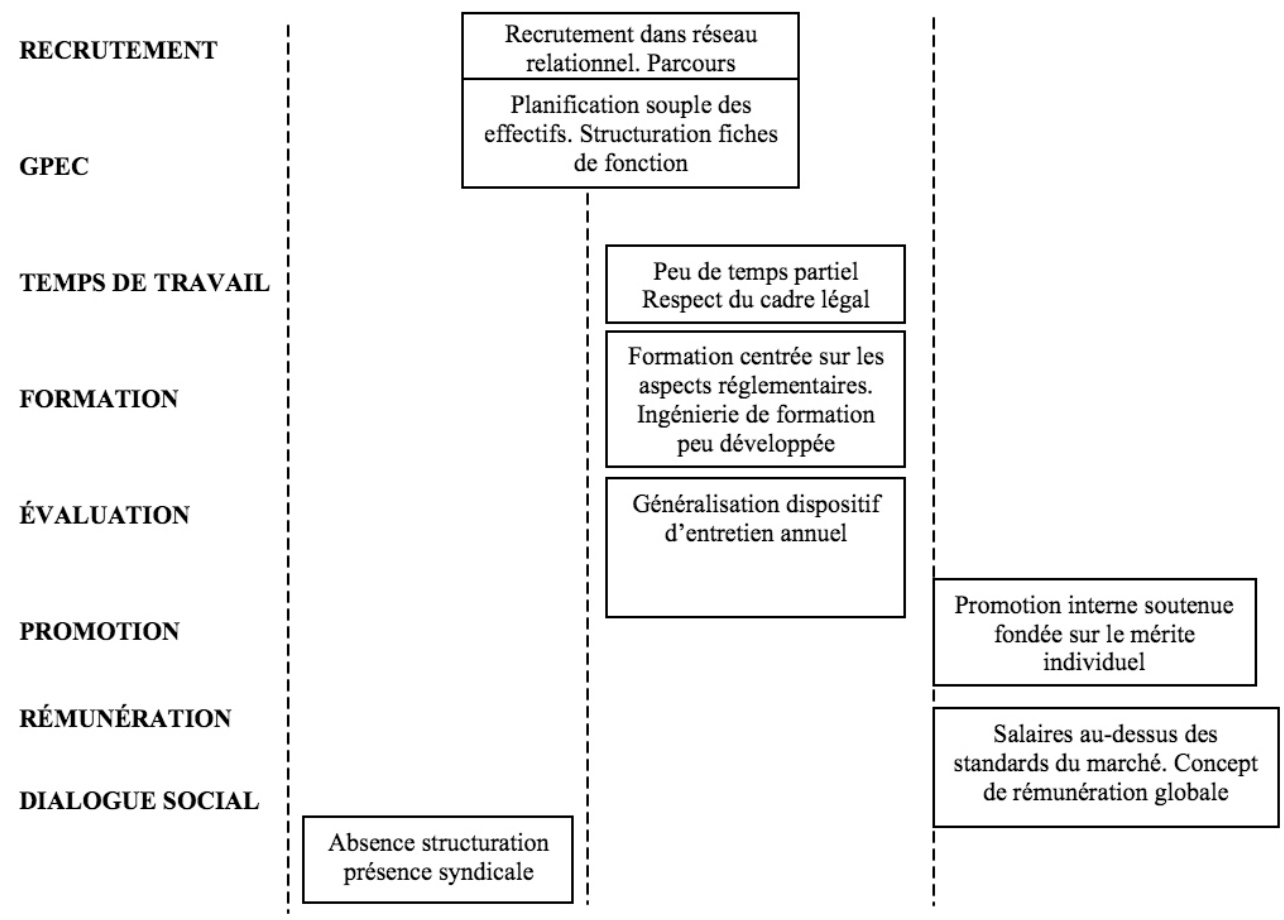

Il semble en l'occurrence que les temporalités variables des décisions ressources humaines et de leurs effets induisent, au final, un double décalage : 1) entre les pratiques RH ellesmêmes (à titre d'exemple, la réorientation du plan de formation est plus aisée à mettre en œuvre qu'un changement culturel) ; 2) entre les pratiques RH et les exigences imposées par la croissance. Cette dé-liaison, ce découplage du système de GRH sont problématiques. Ils

s'impose.

6 Il est intéressant de relever que Defélix et Retour (2003), étudiant le cas d'une PME en croissance, formulent le même constat d'une hybridation du système de GRH. 
mettent en péril la cohérence interne des pratiques de GRH, prévenant l'entreprise de capitaliser sur les effets système, les synergies existant entre pratiques. Or, relèvent Guérin et Wils (2006), c'est moins l'efficacité individuelle de telle ou telle pratique de GRH qui est au fondement de l'avantage concurrentiel que les effets système que leur combinaison induit et leur inscription dans le système social de l'entreprise de façon à se prémunir contre les tentatives d'imitation.

De l'aveu des DRH interviewés, l'investissement dans une démarche de GPEC, qu'il soit déjà incarné ou à l'état de projet, est un puissant vecteur de ré-articulation des pratiques de GRH et contribue à renforcer leur cohérence interne. La GPEC, dans le même temps, est envisagée comme un outil d’alignement de la GRH sur la stratégie d’entreprise, via notamment la réflexion sur l'identification des métiers sensibles. Parlier (2006), pour sa part, voit dans la GPEC un levier d'apprentissage dans un triple registre : apprentissage du changement, de la gestion de projet, de la formalisation. Les PME étudiées confortent par ailleurs l'hypothèse avancée par Parlier (2010) selon laquelle les PME en croissance adopteraient davantage une gestion par les compétences qu'une gestion des compétences. Ainsi, les démarches compétences ne sont pas envisagées comme un simple vecteur de professionnalisation et de rationalisation des processus $\mathrm{RH}$, mais comme une démarche globale s'efforçant d'articuler politique RH, choix d’organisation et choix stratégiques (Zarifian, 2005). Defélix et Retour (2003a, b) assimilent la gestion des compétences dans les PME en croissance à un processus d'apprentissage engageant, d'une part, un travail conséquent sur la sensibilisation des acteurs et l'évolution des représentations, d'autre part, une contextualisation des outils de GPEC pour les fondre dans le moule de la culture interne.

\subsubsection{LA DILUTION DU MODÈLE CULTUREL FONDATEUR}

Les dirigeants tentent de concilier la perpétuation du modèle culturel fondateur et le besoin de structuration induit par la croissance. Cette gestion paradoxale ne va pas de soi et est marquée par le constat récurrent de clivages générationnels : "Les plus anciens sont ceux que nous retrouvons dans les fêtes de l'entreprise. Ils font partie de la famille, ils ont commencé dans cet esprit, ce qui n'est pas le cas des nouveaux. Ces derniers n'ont pas l'esprit de famille" (DRH - NETTOYAGE SUD-EST). La perte de sens ou la déstabilisation identitaire peuvent également toucher les dirigeants comme en atteste ce directeur régional de NETTOYAGE SUD-EST : "En termes de gestion, ce nest pas facile, il faut gérer 8 agences en permanence. Ça manque un peu de visibilité, j’ai plus de projets d'entreprise [...]. Je pense qu’on est obligé de passer par un changement de structure. »

L'étude comparative de cas met ainsi en exergue les tensions que fait peser l'hypercroissance sur la GRH : le besoin de rationalisation s'affronte à la quête de fidélisation des salariés qui suppose des processus davantage individualisés, l'approfondissement des pratiques de GRH s'opère au détriment de leur cohérence interne ; le modèle culturel éprouve ses limites au-delà d'un certain seuil d'effectifs, etc. Quelle grille de lecture adopter à l'égard de ces tensions ? Que révèlent-elles des lignes de force qui structurent l'organisation en hypercroissance? Quels modes de régulation de ces tensions? Ces différents points sont abordés dans la discussion. 


\section{DISCUSSION : LES TENSIONS LIEES Ȧ L'HYPER- CROISSANCE ET LES MODALITÉS DE LEUR RÉGULATION}

\subsection{L'hypercroissance : un processus sous tension}

L'étude comparative de cas réalisée a permis de mettre en relief les tensions et paradoxes que faisait peser la dynamique d'hypercroissance sur l'organisation et plus spécifiquement sur les processus ressources humaines. On doit à Smith et Lewis (2011) une analyse approfondie et un effort de catégorisation des paradoxes organisationnels, particulièrement éclairants pour une approche compréhensive des paradoxes liés à l'hypercroissance. Les auteurs définissent le paradoxe comme "la présence simultanée déléments contradictoires et interdépendants et tendant à perdurer à long terme» (p. 382). Ils identifient quatre paradoxes organisationnels dominants qui, de fait, apparaissent exacerbés par le phénomène d'hypercroissance :

- Le paradoxe de l'apprentissage (learning) renvoie à la tension consacrée par March (1991) entre l'apprentissage par exploitation (l'approfondissement des savoirs existants) et l'apprentissage par exploration (la création de nouveaux savoirs). Cette tension est au cœur de la dynamique même d'hypercroissance : cette dernière crée un besoin de rationalisation et de standardisation des processus ; simultanément une focalisation excessive sur la logique d'exploitation, l'approfondissement des routines actuelles, risque à terme de transformer les compétences fondamentales en rigidités fondamentales (Leonard-Barton, 1992), tout en rendant l'organisation aveugle aux évolutions de son environnement. Les dirigeants de BIO DISTRI témoignent d'une conscience aiguë de cette tension :

"Je perçois toujours l'antinomie entre la perfection de l'organisation et la dynamique de croissance. La dynamique de croissance est fondamentalement désorganisatrice... Par principe, l'organisation prévoit tout et par principe la croissance met toujours l'organisation en retard parce qu'il faut la changer immédiatement » (P.D-G - BIO DISTRI);

"On est une boîte compliquée parce quelle est dans un univers qui est en perpétuelle croissance, donc en désorganisation constante, donc en structuration constante " (DRH - BIO DISTRI);

- Le paradoxe identitaire (belonging) traduit les tensions entre l'individu et le collectif, mais aussi les conflits de rôles et de valeurs qui émergent au fur et à mesure de la croissance de l'organisation. La recherche met ainsi en évidence chez la majorité des acteurs interviewés la crainte d'une dilution du modèle culturel fondateur ;

- Le paradoxe de l'organisant (organizing) exprime les tensions nées de l'accroissement de la complexité organisationnelle, notamment l'articulation délicate entre contrôle et autonomie ou bien encore entre stabilité et changement. Sur le plan de la GRH, la complexité naît de la difficulté dans un régime d'hypercroissance à assurer simultanément l’alignement de la GRH à la stratégie d’entreprise et la cohérence interne des pratiques de GRH ;

- Le paradoxe de la performance (performing) prend acte de la diversité des parties prenantes, internes et externes, et de leurs intérêts, potentiellement contradictoires et conflictuels. Cette problématique est ainsi à l'ouvre dans le cas BIO DISTRI, à travers les relations ambivalentes qu'entretient l'entreprise avec la grande distribution. Le dirigeant estime en effet qu'en investis- 
sant le bio, la grande distribution dénature et dégrade l'image du secteur; dans le même temps, l'entreprise noue un partenariat avec MONOPRIX, considérant qu'il vaut mieux « éduquer » la grande distribution à la commercialisation de produits bio plutôt que les laisser seuls investir ce secteur.

\subsection{Les apports du concept d'ambidextrie pour penser la régulation des tensions liées à l'hypercroissance}

Face aux tensions précédemment évoquées, le concept d’ambidextrie nous semble constituer une piste féconde pour penser les modalités de régulation de ces tensions. Développé il y a quelques années par Tushman et O’Reilly (1996), il désigne cette capacité des organisations et des individus à gérer des processus contradictoires : l'exploitation et l'exploration, la stabilité et le changement, le contrôle et l'autonomie. Cette capacité d'ambidextrie engage un management paradoxal ${ }^{7}$, entendu comme l'aptitude à intégrer simultanément les deux polarités d'une même tension.

L’ambidextrie peut être analysée ici comme une capacité dynamique (Teece, 2007) qui n’est pas une source d'avantage concurrentiel per se, mais favorise les recombinaisons de ressources qui permettront de saisir et d'actualiser les opportunités stratégiques. Le concept d’ambidextrie permet par ailleurs de dépasser une conception de la croissance comme une succession linéaire et prévisible de stades pour l'envisager à l'instar de Levie et Lichtenstein (2010) comme une succession détats dynamiques ${ }^{8}$.

On doit à Simsek et al. (2009) un essai de caractérisation des formes d’ambidextrie organisationnelle opérant à compter de deux dimensions - structurelle et temporelle :

- L'ambidextrie contextuelle traduit la capacité d'une organisation à mener de front logique d'exploitation et logique d'exploration au sein d'une même entité organisationnelle. Ce type d'ambidextrie s'ancre dans la culture, les normes et les valeurs partagées, la création d'un contexte organisationnel permettant aux acteurs d'assumer simultanément les contraintes de l'exploration et de l'exploitation (Gibson et Birkinshaw, 2004). Cela passe entre autres par le développement de la polyvalence, la valorisation de la compétence collective au travers de structures projet, la présence de managers aptes à endosser un large répertoire de rôles (Bierly et Daly, 2007 ; Flyod et Lane, 2000), de raisonner à court terme et à long terme, ou bien encore de gérer des buts conflictuels (Smith et Tushman, 2005);

- L'ambidextrie cyclique consiste à alterner au cours de la trajectoire de croissance des phases dédiées à l'exploitation, l'approfondissement des routines existantes, et des phases dédiées à l'exploration, la création de nouvelles routines. L'ambidextrie cyclique se réfère implicitement au modèle de l'équilibre ponctué qui voit l'organisation alterner des phases courtes de change-

7 À cet égard, le paradoxe ne saurait être confondu avec le dilemme qui consiste à choisir entre deux alternatives.

8 Un état dynamique naît de la tension entre l'environnement et le business model de l'entreprise, qui peut conduire cette dernière à reconfigurer son répertoire de ressources ou à modifier la nature des activités source de création de valeur. 
ment radical (affectant simultanément la structure formelle, les pratiques, les procédures, les systèmes de contrôle et d'allocation des ressources) et des phases longues de relative stabilité (Gersick, 1991). Simsek et al. (2009) observent que l'ambidextrie cyclique est davantage susceptible dêtre engagée par des entreprises ayant ancré leur développement sur l'innovation technologique ;

- L'ambidextrie séparatiste consiste dans l'instauration d'une structure duale ; elle entérine l'hypothèse selon laquelle certaines unités devraient être spécialisées dans l'exploitation et d’autres dans l'exploration. Cette dualité structurelle signifie également une dualité des stratégies, des cultures, des systèmes de contrôle (Benner et Tushman, 2003). L’ambidextrie séparatiste, pour opérer efficacement, suppose une vision stratégique claire et fédératrice, une intégration étroite au niveau de l'encadrement supérieur qui contraste avec la spécialisation étroite des unités organisationnelles et qui permet de mutualiser les expertises ainsi que les informations sur les clients (O'Reilly et Tushman, 2004);

- L'ambidextrie de réseau vise à prémunir l'organisation contre le risque d’obsolescence associé à un développement exclusivement endogène des connaissances (Eisenhardt et Martin, 2000). Elle suppose un partage aigu des connaissances entre les partenaires composant le réseau (Tiwana, 2008) ainsi qu’une capacité combinatoire (Cohen et Lévinthal, 1990) permettant d'intégrer les bases internes et externes de connaissances comme les réseaux relationnels internes et externes (Tempelaar et al., 2008).

Les études de cas mettent en relief la prégnance de l'ambidextrie contextuelle, soit la création d'un contexte organisationnel favorable, permettant aux individus eux-mêmes de faire preuve d'ambidextrie dans leurs activités quotidiennes. Le développement de la polyvalence constitue dans cette optique un levier majeur : "Notre politique maintenant, c'est de privilégier la polyvalence, c'est-à-dire que dans nos derniers recrutements, c'est quelque chose qui a été très impératif» (DRH TEX). La mobilité interne est également activée comme un moyen pour les acteurs délargir leur répertoire de compétences et de comportements : "Il faudrait qu'il y ait davantage de mutations pour que les gens ne prennent pas trop d'habitudes " (DAF NETTOYAGE SUD-EST).

À défaut d'ancrer l'ambidextrie dans les individus eux-mêmes, il peut s'agir d'exploiter leur complémentarité, comme en attestent de nombreux compte rendus à propos de l'équipe de direction : "Le fait dêtre à trois, je pense que jai plus de chance de ne pas me tromper et de mieux percevoir » (D-G TEX); "Le but est d'être à plusieurs pour prendre une décision. Il ne faut jamais être seul pour prendre une décision importante »(P.D-G NETTOYAGE SUD-EST).

L'ambidextrie cyclique ou temporelle semble inhérente à la trajectoire même d'hypercroissance, dont Julien, St-Jean et Audet (2006) notent la nature disruptive, alternant des phases courtes de changements brutaux et de transformations organisationnelles profondes et des phases plus durables de relative stabilité. Chacune des études de cas réalisées révèle de tels moments de rupture, qu'il s'agisse de l'entrée d'un fonds d'investissement qui devient actionnaire majoritaire (BIO DISTRI) ou de l'obtention d'une certification qualité (NETTOYAGE SUD EST).

L'ambidextrie de réseau, pour sa part, semble faire l'objet d'une prise de conscience aiguë dans les monographies réalisées. Dans le cas TEX, elle consiste à s'appuyer sur les fournisseurs pour alimenter la dynamique d'exploration (« Nos fournisseurs, nos quatre principaux fournisseurs, sont des partenaires pour l'évolution » - DRH TEX) ou à envisager la croissance externe comme un 
moyen pour l'organisation de préempter les compétences qui lui font défaut. Comme le souligne la revue de littérature, l'ambidextrie de réseau, pour réaliser pleinement son potentiel, suppose une densification des liens entre partenaires et la structuration de dispositifs de transferts interorganisationnels de connaissances. Le D-G de NETTOYAGE SUD-EST déplore à cet égard l'absence de véritable structure d'intégration pour piloter les opérations de croissance externe : "On sait acheter, on sait valoriser, mais on ne sait pas intégrer. Nous navons pas de structure d'acquisition, c'est ce qui manque [...] Il nous faut pour les années à venir, si l'on veut continuer la croissance externe, monter une équipe fusion/acquisition qui soit compétente, avec en son sein des financiers, des juristes, du marketing, c’est-à-dire des pôles de compétences spécialisés » (DAF, TEX).

Les études de cas, enfin, mettent en exergue une autre modalité d'ambidextrie : celle qui consiste à jouer sur la variété des profils, la diversité des expériences, comme en atteste la coexistence au sein de BIO DISTRI d'une population de salariés " fidèles » convaincus par la dimension militante du projet et d'une population de salariés «mercenaires» au profil gestionnaire plus affirmé : "Pour moi il y a les fidèles et puis les mercenaires, ceux qui viennent juste pour leur CV, qui pillent la boite et qui sén vont. On en a quand même besoin à un certain moment, ce sont des personnes qui vont être efficaces et qui apportent un œil nouveau... la boite ne peut pas que garder des fils de producteurs qui ont du cour et du bon sens... Ce qui est difficile pour BIO DISTRI, c'est d'arriver à conjuguer les deux milieux » (DRH).

Quelles que soient les formes d'ambidextrie à l'œuvre, la présence d'une vision stratégique claire, ambitieuse, incarnée, semble constituer un élément crucial dans la construction d’organisations ambidextres (O’Reilly et Tushman, 2004).

Au terme de notre analyse, la contribution de cet article semble pouvoir s'inscrire dans un triple registre :

- D'un point de vue théorique, elle souligne l'intérêt d'une approche par les tensions ou le paradoxal pour appréhender la relation entre GRH et hypercroissance là où les approches traditionnelles par les stades de croissance apparaissent trop déterministes et souffrent d'un manque de validation empirique ;

- Elle montre comment face aux tensions induites par l'hypercroissance les PME mobilisent une capacité d'ambidextrie multiforme (tout en donnant à voir un nouveau type d'ambidextrie que nous qualifions d' « humaine », jouant sur la diversité des profils);

- Sur un plan empirique, la contribution met également en relief la spécificité des problématiques et pratiques de GRH en hypercroissance, alertant en particulier sur le risque de déliaison, de découplage du système de GRH ;

- D'un point de vue méthodologique enfin, on soulignera l'intérêt qu'il y a à s'appuyer sur un matériau longitudinal à l'égard d'un objet - les PME en hypercroissance où les travaux empiriques restent rares.

\section{CONCLUSION}

L'ambition de cette contribution n'est pas de définir les «bonnes pratiques » de GRH susceptibles de soutenir une dynamique d'hypercroissance en PME. Semblable approche relève en 
effet selon nous d'une double méprise : 1) elle sous-estime le caractère contingent et nécessairement contextualisé des politiques et des pratiques de GRH ;2) elle minore la part d'aléa ou de chance que comporte toute dynamique d'hypercroissance. Les approches fondées sur les seuils ou stades de croissance ne semblent par ailleurs pas validées empiriquement.

Notre ambition, dès lors, est plus modeste : il s'agit de repérer les points de tension que fait peser le phénomène d'hypercroissance sur la fonction ressources humaines et d'envisager les leviers de régulation de ces tensions. Ces tensions sont, entre autres, liées au besoin de rationalisation des pratiques de GRH et à la nécessité d'un partage plus aigu de la fonction RH avec l'encadrement, au risque de découplage des pratiques de GRH et leur désalignement avec la stratégie, à la dilution du modèle culturel fondateur induite par le phénomène d'hypercroissance. L'étude comparative de cas réalisée montre à cet égard l'importance de la GPEC comme vecteur de réarticulation des pratiques de GRH, mais aussi comme source d'apprentissage : de la formalisation, du changement, de la gestion de projet. À un niveau plus agrégé, notre travail souligne l'importance de la capacité d'ambidextrie pour soutenir l'hypercroissance. L'étude comparative de cas met de ce point de vue en relief le poids de l'ambidextrie contextuelle dans les PME en hypercroissance, tout en donnant à voir une nouvelle forme d'ambidextrie que nous qualifions d' « humaine ". Cependant, il conviendrait sans doute à terme d'interroger la façon dont ces différentes formes d'ambidextrie s'articulent dans un contexte d'hypercroissance (Raisch et al., 2009).

AnNexe 1. Présentation SynthétiQue des études de CaS ET DU Matériau EMPIRIQUe COLLECTÉ

\begin{tabular}{|c|c|c|c|}
\hline & BIO DISTRI & $\begin{array}{l}\text { NETTOYAGE } \\
\text { SUD-EST }\end{array}$ & TEX \\
\hline \multicolumn{4}{|c|}{ Matériau empirique collecté } \\
\hline Nombre entretiens & 8 & 5 & 6 \\
\hline Acteurs rencontrés & $\begin{array}{l}\text { P.D-G } \\
\text { Directeur administratif } \\
\text { et financier } \\
\text { Secrétaire générale } \\
\text { DRH } \\
\text { Contrôleur de gestion } \\
\text { Responsable logistique } \\
\text { Responsable qualité }\end{array}$ & $\begin{array}{l}\text { P.D-G fondateur } \\
\text { Directeur général } \\
\text { Directeur régional } \\
\text { Directrice des } \\
\text { ressources humaines } \\
\text { Secrétaire générale }\end{array}$ & $\begin{array}{l}\text { Directeur général } \\
\text { DRH } \\
\text { DAF } \\
\text { Directrice commerciale } \\
\text { Directeur des } \\
\text { opérations et de } \\
\text { la production } \\
\text { Client distributeur } \\
\text { indien }\end{array}$ \\
\hline \multicolumn{4}{|l|}{ Fiche signalétique } \\
\hline Secteur d'activité & $\begin{array}{l}\text { Metteur en marché } \\
\text { fruits et légumes bio }\end{array}$ & $\begin{array}{l}\text { Nettoyage et propreté } \\
\text { tous locaux }\end{array}$ & $\begin{array}{l}\text { Fabrication de } \\
\text { thermocollants }\end{array}$ \\
\hline Date création & 1987 & 1967 & 1983 \\
\hline Siège & Vaucluse (France) & $\begin{array}{l}\text { Hautes-Alpes } \\
\text { (France) }\end{array}$ & Loire (France) \\
\hline Effectifs 2008 & 220 & $\begin{array}{l}1300 \text { (617 équivalent } \\
\text { temps plein) }\end{array}$ & 49 \\
\hline Label/Certification & Oui & Oui & Oui \\
\hline
\end{tabular}




\begin{tabular}{|c|c|c|c|}
\hline \multicolumn{4}{|c|}{ Dynamique générale de croissance } \\
\hline $\begin{array}{l}\text { Phases/stades de } \\
\text { croissance }\end{array}$ & 3 & 4 & 3 \\
\hline Hypercroissance & $20 \% /$ an $(2005 / 2009)$ & $26 \% /$ an $(2000 / 2004)$ & $28 \% /$ an $(2002 / 2006)$ \\
\hline $\begin{array}{l}\text { Objectif } \\
\text { général }\end{array}$ & $\begin{array}{l}\text { Pérenniser et légitimer } \\
\text { l'activité } \\
\text { Définir les standards du } \\
\text { marché }\end{array}$ & $\begin{array}{l}\text { S'affirmer comme } \\
\text { un acteur régional } \\
\text { important } \\
\text { Croître en saisissant les } \\
\text { opportunités }\end{array}$ & $\begin{array}{l}\text { S'affirmer comme un } \\
\text { des principaux acteurs } \\
\text { nationaux sur un } \\
\text { marché de niche }\end{array}$ \\
\hline $\begin{array}{l}\text { Positionnement } \\
\text { stratégique }\end{array}$ & $\begin{array}{l}\text { Stratégie de } \\
\text { différenciation fondée } \\
\text { sur l'innovation et } \\
\text { la proximité avec les } \\
\text { fournisseurs et clients }\end{array}$ & $\begin{array}{l}\text { Stratégie de } \\
\text { différenciation fondée } \\
\text { sur la qualité des } \\
\text { prestations et une } \\
\text { gamme de services } \\
\text { associés }\end{array}$ & $\begin{array}{l}\text { Stratégie de } \\
\text { différenciation sur } \\
\text { l'innovation, l'étendue } \\
\text { et la personnalisation } \\
\text { de l'offre }\end{array}$ \\
\hline $\begin{array}{l}\text { Mode de croissance } \\
\text { privilégié }\end{array}$ & Croissance externe & Croissance externe & $\begin{array}{l}\text { Croissance interne } \\
\text { dominante }\end{array}$ \\
\hline
\end{tabular}

\section{BIBLIOGRAPHIE}

Autier F. et Picq T. (2002), Gestion stratégique des ressources humaines : une situation paradoxale?, Revue de Gestion des Ressources Humaines, juillet-août-septembre, 45, 6-19.

BARNEY J. (1991), Firm resources and sustained competitive advantage, Journal of Management, 17, 1, 99-120.

BARRINGER B.R., JoNES F.F. et LEwIS P.S. (1998), A qualitative study of the management practices of rapid growth firms mitigate the managerial capacity problem, Journal of Developmental Entrepreneurship, $3,2,97-122$.

Benner M.J. et Tushman M.L. (2003), Exploitation, exploration and process management : the productivity dilemma revisited, Academy of Management Review, 28, 2, 238-256.

Besseyre Des Horts C.H. (2002), La GRH est-elle créatrice de valeur? L'exemple de la e-RH, Revue de Gestion des Ressources Humaines, octobre-décembre, 46, 2-16.

Betbèze J.P. et Saint-Étienne C. (2006), Une stratégie PME pour la France, Rapport du Conseil d'Analyse Économique, 61, 165 p.

BIERLY P.E. et DALY P.S. (2007), Alternative knowledge strategies, competitive environment, and organizational performance in small manufacturing firms, Entrepreneurship Theory et Practice, 31, 4, 493-516.

Bootz J.P., Schenk E. et Sonntag M. (2010), Gestion stratégique des compétences en PME : les enseignements d'une recherche-action, Larchive ouverte HAL-SHS, <http://halshs.archives-ouvertes.fr/ halshs-00456007/en/>, consulté le 14 novembre 2013.

Chanut-Guieu C. et Guieu G. (2011), Stratégie et structuration des trajectoires d'hypercroissance des PME. Une étude comparative de cas, Management et Avenir, 3, 43, 37-56.

Charreire S. et Durieux F. (2003), Explorer et tester : deux voies pour la recherche, dans Méthodes de recherche en management, R.A. Thiétart (dir.), Paris, Dunod, 57-81. 
Cohen W.M. et Levinthal D.A. (1990), Absorptive capacity : a new perspective on learning and innovation, Administrative Science Quaterly, 35, 1, 128-152.

Defélix C. et Retour D. (2003a), La gestion des compétences dans la stratégie de croissance d'une PME innovante : le cas Mikrotech, Revue internationale PME, 16, 3-4, 31-52.

DefÉLix C. et Retour D. (2003b), La gestion des compétences comme processus d’apprentissage : une analyse longitudinale dans deux PME en croissance, dans Gérer les compétences. Des instruments aux processus, A. Klarsfeld et E. Oiry (dir.), Paris, Vuibert, 89-105.

Dejoux C. (2008), Gestion des compétences et GPEC, $1^{\text {re }}$ édition, Paris, Dunod (Les Topos), 127 p.

Delmar F., Davidsson P. et Gartner W.B. (2003), Arriving at the high-growth firm, Journal of Business Venturing, 18, 2, 189-216.

EISEnhardt K. et Martin J.A. (2000), Dynamic capabilities : what are they ?, Strategic Management Journal, 21, 10-11, 1105-1121.

FLYOD S. et LANE P. (2000), Strategizing throughout the organization : managing role conflict in strategic renewal, Academy of Management Review, 25, 1, 154-177.

GASSE Y. (1996), Croissance et gestion de l'entreprise nouvelle, $3^{e}$ Congrès international francophone en entrepreneuriat et PME, Université du Québec à Trois-Rivières, 23-25 octobre.

Gersick C.J.G. (1991), Revolutionary change theories : a multilevel exploration of the punctuated equilibrium paradigm, Academy of Management Review, 16, 1, 10-36.

Gibson C. et Birkinshaw J. (2004), The antecedents, consequences and mediating role of organizational ambidextry, Academy of Management Journal, 47, 2, 209-226.

Greiner L.E. (1972), Evolution and revolution as organizations grow, Harvard Business Review, 50, 4, $37-46$.

GuéRIN G. et WiLs T. (2006), Lalignement stratégique de la gestion des ressources humaines, dans Encyclopédie de gestion des ressources humaines, $2^{\mathrm{e}}$ édition, J. Allouche (dir.), Paris, Vuibert, 16-25.

JANSSEN F. (2005), La conceptualisation de la croissance : l'emploi et le chiffre d'affaires sont-ils des représentations interchangeables d'un même phénomène?, Gestion 2000, 22, 6, 267-291.

JaOuen A. et Torrès O. (2008), Les très petites entreprises : un management de proximité, Paris, HermèsLavoisier, $380 \mathrm{p}$.

Julien P.-A., St-Jean E. et Audet J. (2006), Les facteurs de discontinuité des PME à forte croissance, $8^{e}$ Congrès international francophone en entrepreneuriat et PME, Haute École de gestion, Fribourg, Suisse, 25-27 octobre.

JuLien P.-A. (2001), Les PME à forte croissance et la métaphore du Jazz. Comment gérer l'improvisation organisationnelle de façon cohérente, Revue internationale PME, 14, 3-4, 129-162.

Julien P.-A. (dir.), Luc D., Carrier C., Desaulniers L. et Martineau Y. (2002), Les PME à forte croissance, Sainte-Foy, Presses de l'Université du Québec, 243 p.

Koenig G. (1993), Production de la connaissance et constitution des pratiques organisationnelles, Revue de gestion des ressources humaines, 9, novembre, 4-17.

Lado A. et WiLson M.C. (1994), Human resource systems and sustained competitive advantage : a competency-based perspective, Academy of Management Review, 19, 4, 699-727. 
LeONARD-BARTON D. (1992), Core capabilities and core rigidities : a paradox in managing new product development, Strategic Management Journal, 13, 1, 111-125.

Levie J. et Lichtenstein B. (2010), A terminal assesment : introducing a dynamic stages approach to entrepreneurship, Entrepreneurship and Practice, 34, 2, 317-350.

Louart P. et Vilette M.A. (2010), La GRH dans les PME, Paris, Vuibert, 416 p.

Mahé de Boislandelle H. (1988), Gestion des ressources humaines dans les PME, Paris, Economica, $322 \mathrm{p}$.

MARCH J. (1991), Exploration and exploitation in organizational learning, Organization Science, 2, 1, 71-87.

Marchesnay M. (1991), La PME : une gestion spécifique ?, Économie rurale, 206, novembre-décembre, 11-17.

Mustar P. (2002), Les PME à forte croissance et l'emploi, Rapport de recherche de l'OCDE, 149 p.

Nicholls-Nixon C. (2005), Rapid growth and high performance : the entrepreneur's impossible dream, Academy of Management Executive, 19, 1, 1-13.

Nizet J. et Pichault F. (2000), Les pratiques de gestion des ressources humaines, Paris, Éditions du Seuil (Points Seuil), $322 \mathrm{p}$.

O'Reilly C.A. et Tushman M.L. (2004), The ambidextrous organization, Harvard Business Review, 82, $4,74-82$.

Paradas A. (2010), Les contraintes de recrutement dans les PME, dans La GRH dans les PME, P. Louart et M.A. Vilette (dir.), Paris, Vuibert, 223-236.

PARLIER M. (2006), Les ressources humaines dans les petites et moyennes entreprises, dans Encyclopédie de gestion des ressources humaines, $2^{\mathrm{e}}$ édition, J. Allouche (dir.), Paris, Vuibert, 961-969.

Parlier M. (2010), Gérer les compétences en PME, dans La GRH dans les PME, P. Louart et M.A. Vilette (dir.), Paris, Vuibert, 175-199.

Penrose E.T. (1959), The theory of the growth of the firm, New York, John Wiley et Sons, $272 \mathrm{p}$.

Pettigrew A. (1985), Contextualist research and the study of organizational change processes, dans Doing research that is useful for theory and practice, E. Lawler (dir.), San Francisco, Jossey-Bass, 53-72.

PiCARD C. (2006), Les gazelles en France, Document de travail de la direction des études et synthèses économiques, INSEE.

QUADER M.S. (2007), Human resource management issues as growth barriers in professional service firm SME's, Journal of Services Research, 7, 2, 115-161.

Raisch S., Birkinshaw J., Probst G. et Tushman M.L. (2009), Organizational ambidexterity : balancing exploitation and exploration for sustained performance, Organization Science, 20, 4, 685-695.

Simsex Z., Heavy C., Veiga J.F. et Souder D. (2009), A typology for aligning organizational ambidexterity's conceptualizations, antecedents and outcomes, Journal of Management Studies, 46, 5, 864-894.

SмIтн W.K. et LEwis M.W. (2011), Toward a theory of paradox : a dynamic equilibrium model of organizing, Academy of Management Review, 36, 2, 381-403.

Smith W.K. et Tushman M.L. (2005), Managing strategic contradictions. A top management model for managing innovation streams, Organization Science, 16, 5, 522-536. 
Teece D.J. (2007), Explicating dynamic capabilities : the nature and microfoundations of (sustainable) enterprise performance, Strategic Management Journal, 28, 13, 1319-1350.

Tempelaar M., Jansen J.J.P. et Van Den Bosch F.A.J. (2008), Knowing your clients : the joint effect of client and internal social capital on organizational ambidexterity, Conférence annuelle sur le management stratégique des sociétés, Cologne, Allemagne, 12-15 octobre.

Tiwana A. (2008), Do bridging ties complement strong ties? An empirical examination of alliance ambidexterity, Strategic Management Journal, 29, 3, 251-272.

Tushman M. et O’Reilly C. (1996), Ambidextruous organizations : managing evolutionary and revolutionary change, California Management Review, 38, 4, 8-30.

Wright P.M., McMahan G.C. et McWilliams A. (1994), Human resources and sustained competitive advantage : a resource-based perspective, International Journal of Human Resource Management, 5, 2, 301-326.

Zarifian P. (2005), Compétences et stratégie de l'entreprise, Paris, Éditions Liaisons, 244 p. 